\title{
Protective effects of ethyl pyruvate on lipopolysaccharide-induced acute lung injury through inhibition of autophagy in neutrophils
}

\author{
QINGTENG ZHU ${ }^{1 *}$, HUI WANG ${ }^{2 *}$, HAIRONG WANG $^{1}$, YONG LUO $^{3}$, \\ YANG YU $^{1}$, QIRONG DU ${ }^{1}$, AIHUA FEI ${ }^{1}$ and SHUMING PAN ${ }^{1}$
}

\author{
${ }^{1}$ Department of Emergency Medicine, Xinhua Hospital Affiliated to Shanghai Jiao Tong University \\ School of Medicine, Shanghai 200092; ${ }^{2}$ Department of Respiratory Medicine, Putuo Hospital Affiliated to \\ Shanghai University of Traditional Chinese Medicine, Shanghai 200062; ${ }^{3}$ Department of Respiratory Medicine, \\ Xinhua Hospital Affiliated to Shanghai Jiao Tong University School of Medicine, Shanghai 200092, P.R. China
}

Received November 21, 2015; Accepted November 24, 2016

DOI: $10.3892 / \mathrm{mmr} .2017 .6118$

\begin{abstract}
Among a number of clinical factors, bacterial infection is one of the most common causes of acute lung injury (ALI), a serious complication that carries a high risk of mortality ( $40 \%)$. During the process of ALI, intense local and systemic inflammation is elicited, which exacerbates the injury. Neutrophil infiltration into airspace is observed in early stage of ALI, and is required for the full development of ALI through an array of mechanisms, including the release of granule contents and the production of pro-inflammatory cytokines, due to the overactivation of complement and cytokines. The present study noted that ethyl pyruvate alleviated ALI in lipopolysaccharide (LPS)-induced ALI mice. Increased autophagy in neutrophils from ALI mice was observed, while ethyl pyruvate diminished autophagy in neutrophils and constrained granule release, and therefore myeloperoxidase (MPO) in bronchoalveolar lavage fluid and the production of proinflammatory cytokines. Using neutrophil cells, it was identified that autophagy was required for neutrophil activation and granule release, and that ethyl pyruvate caused neutrophil autophagy, leading to the restriction of granule release, and thus contributing to the mitigation of ALI. If autophagy was obviated through knockdown of key regulator of autophagy
\end{abstract}

Correspondence to: Dr Aihua Fei or Dr Shuming Pan, Department of Emergency Medicine, Xinhua Hospital Affiliated to Shanghai Jiao Tong University School of Medicine, 1665 Kongjiang Road, Shanghai 200092, P.R. China

E-mail: aihuafei@aliyun.com

E-mail: shumingpan@aliyun.com

*Contributed equally

Abbreviations: ALI, acute lung injury; BAL, branchoalveolar lavage; fMLP, N-Formyl-Met-Leu-Phe; IL-6, interleukin-6; LPS, lipopolysaccharide; MPO, myeloperoxidase; TNF- $\alpha$, tumor necrosis factor- $\alpha$

Key words: ethyl pyruvate, acute lung injury, neutrophil, autophagy
Atg5, the effects of ethyl pyruvate on granule release by neutrophils disappeared. Taken together, the results demonstrated that ethyl pyruvate alleviates ALI through inhibition of autophagy-induced granule release by neutrophils, and this mechanism suggested a novel potential therapeutic target in autophagy regulation for ALI.

\section{Introduction}

Numerous clinical events, including sepsis, trauma, transient ischemia and reperfusion, cause acute lung injury (ALI) (1). Sepsis, as a result of severe infection, is one of the most common clinical causes of ALI and leads to a high mortality of $60-80 \%$ (2). Although the management of critically ill patients is improving, the mortality rate remains $\sim 40 \%$, and survivors often do not return to a normal life (3). In both immunocompromised and immunocompetent individuals, bacterial pneumonia is a leading cause of mortality from ALI. During the process, neutrophil infiltration is the first event, followed by large amount of proinflammatory cytokines and proteases, including myeloperoxidase (MPO), which are the main mediators in the causes of serious lung injury. Presently, only a few pharmacological treatments are available for lipopolysaccharide (LPS)-induced ALI; the inhibition of inflammation or the production of antioxidative effects.

Ethyl pyruvate is a stable and simple lipophilic ester derived from the endogenous metabolite pyruvate and has been demonstrated to protect against inflammation and to mitigate organ dysfunction in several animal models of various disorders, including severe sepsis, burns and acute pancreatitis. Ethyl pyruvate has also been demonstrated to possess a protective effect on ALI, probably through the inhibition of the mitogen-activated protein kinase (MAPK) pathway in lungs $(4,5)$. However, these results simply reveal the general ameliorative effect of ethyl pyruvate with no cellular target and therefore, more data are required to elucidate the underlying mechanism.

Previous studies (6-9) have demonstrated that neutrophils extensively accumulate in the lung during the initial stage of ALI, which substantially contributes to the severity of this disorder in human and animal models by several mechanisms: 
i) The release of granule components, including serine proteases, matrix metalloproteinases and lactoferrin; ii) the generation of reactive oxygen species; and iii) the formation of neutrophil extracellular traps, thus making neutrophils a potential target for ALI attenuation. Different therapeutic approaches have been developed against neutrophil infiltration, the releasing of granule components and the induction of apoptosis.

Autophagy is a constitutive regulatory means of cellular homeostasis involved in diverse physiological and pathological events (10-15). Autophagy has been demonstrated to be important for bacterial infection and inflammation (16-18), for example, autophagy in macrophages is important for the maturation, activation, polarization and regulation of cytokine production (19-23). Several mechanisms of anti-inflammation by autophagy have been proposed, including clearing the cytoplasm of non-functional organelles (e.g., mitochondria), and removing the aggregated inflammasome structure (16). However, few studies exist on the regulation of neutrophils by autophagy, and recently it was demonstrated that autophagy deficiency reduced neutrophil degranulation (24). Whether ethyl pyruvate contributes to neutrophil autophagy in ALI remains to be elucidated.

The present study aimed to confirm the protective effects of ethyl pyruvate on ALI in vivo using an LPS-induced mouse model, and to further elucidate the underlying mechanism. The data demonstrated that neutrophils infiltrated into airspace during ALI experience increased autophagy, which is required for granule release, while ethyl pyruvate inhibited autophagy in neutrophils, and decreased granule release, thus attenuating lung injury in ALI. This novel mechanism illuminates the role of ethyl pyruvate in ALI, and provides a basis from which to develop a novel therapeutic approach with autophagy as a target.

\section{Materials and methods}

Reagents and antibodies. Ethyl pyruvate, lipopolysaccharide and N-Formyl-Met-Leu-Phe were obtained from Sigma-Aldrich (Merck Millipore, Darmstadt, Germany). Antibodies against LC3 (cat. no. 4108S, 1:2,000), Becn1 (cat. no. 3738S, 1:2,000), ATG5 (cat. no. 12994, 1:2,000), and $\beta$-actin (cat. no. 4970S, 1:2,000) were obtained from Cell Signaling Technology, Inc. (Danvers, MA, USA). PE-Ly6G [1A8] and APC-Gr1 [RB6-8C5] were purchased from BioLegend, Inc. (San Diego, CA, USA). ELISA Kits for tumor necrosis factor- $\alpha$ (TNF- $\alpha$ ), interleukin-6 (IL-6) and MPO were purchased from R\&D Systems, Inc. (Minneapolis, MN, USA).

Experimental model. C57BL/6 male mice ( $\mathrm{n}=56 ; 8$ weeks old) used for preparing ALI models were obtained from the Shanghai Laboratory Animal Center of the Chinese Academy of Sciences (Shanghai, China), and maintained under specific pathogen-free conditions at $22^{\circ} \mathrm{C}, 50 \%$ humidity and a $12 \mathrm{~h}$ light/dark cycle, with free access to food and sterile water. The animals were weighed, injected intratracheally with LPS $(5 \mathrm{mg} / \mathrm{kg}$ ) or vehicle (phosphate-buffered saline; PBS) and euthanized with $\mathrm{CO}_{2}$ at $2 \mathrm{l} / \mathrm{min}$ in a closed box of $\sim 10 \mathrm{l}$ volume. The concentration of $\mathrm{CO}_{2}$ was gradually increased to $70 \%$ within $\sim 4 \mathrm{~min}$ ) for $15 \mathrm{~min}$, and mortality was confirmed upon no response to hind limb pinching at 4 or $24 \mathrm{~h}$ following injection. All experiments were performed in accordance with the guidelines of, and with the approval of, the Animal Care and Usage Committee of Xinhua Hospital, Shanghai Jiao Tong University School of Medicine (Shanghai, China).

Histopathology. Lung samples were fixed in $10 \%$ formalin, sectioned and dehydrated through 70, 80 and 95\% alcohol, 45 min each, followed by 3 changes of $100 \%$ alcohol, $1 \mathrm{~h}$ each, followed by embedding in paraffin wax. Tissue blocks were sectioned to $5 \mathrm{~mm}$, transferred to glass slides and stained with hematoxylin and eosin. Morphological examinations were performed using light microscopy and images were captured.

Cell culture and transfection. The murine myeloid cell line, 32Dcl3 (CRL-11346), was obtained from American Type Culture Collection (Manassas, VA, USA), and cultured in RPMI-1640 medium supplemented with $10 \%$ fetal bovine serum. The $32 \mathrm{Dcl} 3$ cells were transfected with Lipofectamine 3000 (Invitrogen; Thermo Fisher Scientific, Inc., Waltham, MA, USA), according to the manufacturer's protocol.

Determination of cytokines and MPO in murine bronchoalveolar lavage fluid (BALF) by ELISA. TNF- $\alpha$, IL-6, and MPO in murine BALF were determined by sandwich ELISA (R\&D Systems, Inc.), according to the manufacturer's protocol.

Immunoblotting. Immunoblotting was performed as described previously (17). Briefly, cells with or without treatment were collected and lysed in radioimmunoprecipitation assay buffer (Beyotime Institute of Biotechnology, Haimen, China). Following brief vortexing and rotation, cell lysates were separated by SDS-PAGE and transferred onto PVDF membranes. The membranes were blocked with 5\% non-fat milk in PBS for $30 \mathrm{~min}$ at room temperature and were subsequently incubated with appropriate antibody in PBS with $0.5 \%$ non-fat milk for $2 \mathrm{~h}$ at room temperature. Following washing in PBS/Tween-20, the membranes were incubated for $1 \mathrm{~h}$ with horseradish peroxidase-conjugated secondary antibody. Bands were detected with enhanced chemiluminescence plus detection reagents (Amersham Pharmacia Biotech, Piscataway, NJ, USA).

Autophagy analyses. Autophagy was analyzed by immunoblotting or fluorescence microscopy, as described previously (25). Briefly, cell lysates were immunoblotted with rabbit monoclonal anti-microtubule-associated protein 1A/1B-light chain 3 (LC3) antibody (Cell Signaling Technology, Inc.; cat. no. 4108S) at 1:2,000 dilution, followed by incubation with HRP-linked anti-rabbit secondary antibody (cat. no. 7074S; Cell Signaling Technology, Inc.) to monitor LC3-II generated during the formation of autophagosomes.

Pulmonary leukocyte isolation. Animals were euthanized with $70 \% \mathrm{CO}_{2}$ following approved protocols, and cells from individual mice were collected for further experiments. BAL was collected, and the cells were dispersed by repetitive suction through a $10 \mathrm{ml}$ syringe and centrifuged at $400 \mathrm{x} g$ for $10 \mathrm{~min}$ at room temperature. Pellets were resuspended in 


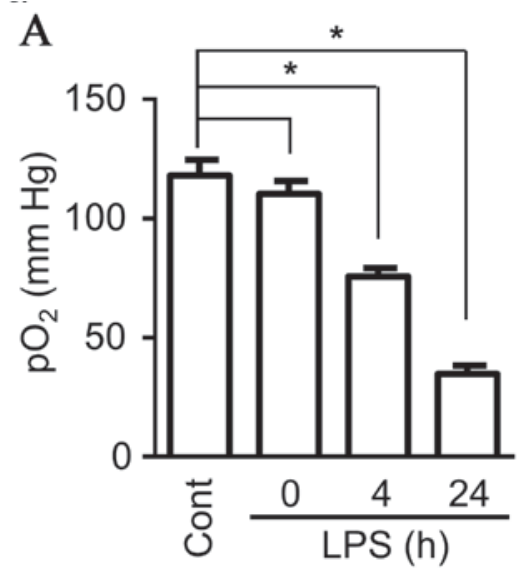

C

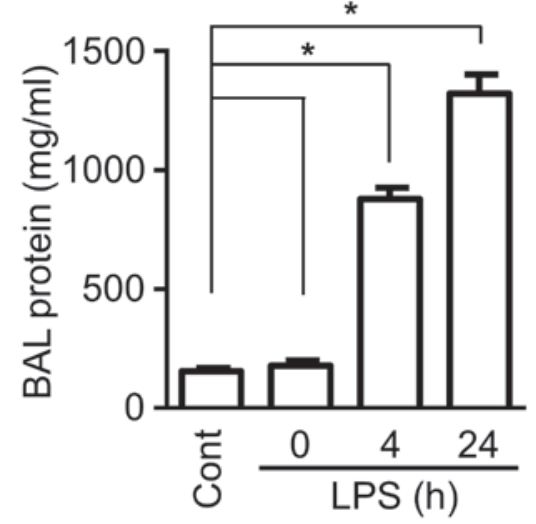

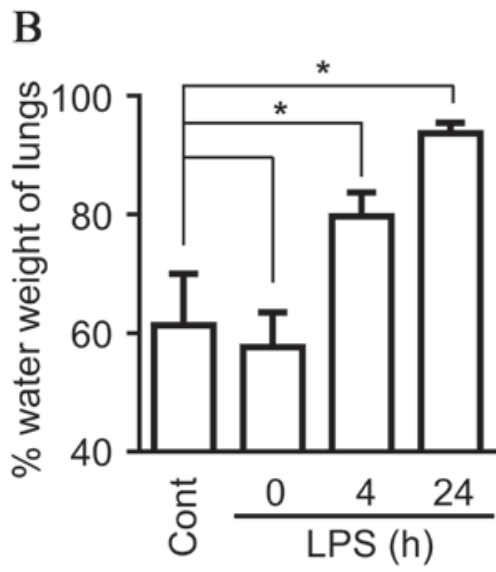

$\mathrm{D}$

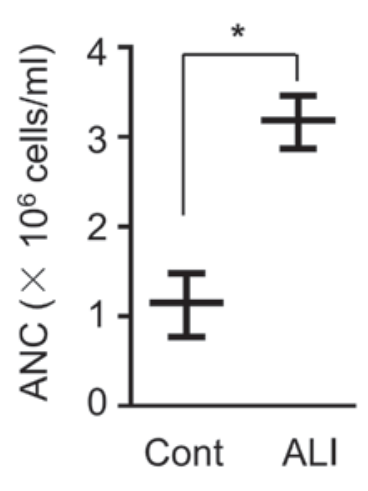

Figure 1. Accumulated neutrophils in BALF from intestinal LPS-induced ALI in mice. Eight-week old C57BL/6 male mice (6 mice per group) were injected intratracheally with LPS $\left(5 \mathrm{mg} / \mathrm{kg}\right.$ ) at 4 or $24 \mathrm{~h}$. (A) Arterial blood $\mathrm{pO}_{2}$ was identified. (B) Mouse lungs were removed, and percent water content measured. BALF was collected, and (C) protein contents and (D) ANC were measured. The data are presented as the mean \pm standard deviation of three independent experiments ( $\mathrm{P}<0.05)$. BALF, bronchoalveolar lavage fluid; LPS, lipopolysaccharide; ANC, absolute neutrophil count; ALI, acute lung injury; Cont, control.

$1 \mathrm{ml}$ sterile double-distilled $\mathrm{H}_{2} \mathrm{O}$ to lyse red blood cells and centrifuged, as before. The pellets were resuspended in $5 \mathrm{ml}$ complete medium or PBS.

Flow cytometric analysis. BALF cells and lung leukocytes were assessed using flow cytometry. BAL cells $(50,000$ cells) in $100 \mu \mathrm{l}$ flow assay buffer were incubated with PE-Ly6G [1A8] (\#127607) and APC-Gr1 [RB6-8C5] (\#108411) (Biolegend, San Diego, CA, USA). Cells were washed again, resuspended in $3 \%$ paraformaldehyde and analyzed using a FACS Caliber flow cytometer (BD Bioscience, San Jose, CA, USA).

Statistics. Two-tailed Student's t-test or one-way analysis of variance were used for statistical analyses with GraphPad Prism 6 (GraphPad, San Diego, CA, USA). Quantitated data from at least 3 independent experiments were shown as the mean \pm standard error of the mean. $\mathrm{P}<0.05$ was considered to indicate a statistically significant difference.

\section{Results}

Accumulated neutrophils in BALF from LPS-induced ALI in mice. Sepsis is one of the most common clinical causes of ALI/acute respiratory distress syndrome (ARDS), so in order to further research on the mechanism underlying the acute lung injury, an LPS-induced lung injury model mouse was
established.LPS, a component of the cell wall of Gram-negative bacteria, can induce severe inflammatory responses, and intratracheal administration of LPS has gained wide acceptance as a clinically relevant model of ALI/ARDS in mice. During the development of ALI, changes in breathing patterns are observed, accompanied by lower blood oxygenation (Fig. 1A; known indicators of ALI) $(26,27)$. In addition, more intravascular content leakage was observed due to dysfunction of the capillary walls, including increased lung water content (Fig. 1B), as well as more protein and more blood granulocytes present in BALF (Fig. 1C and D). Flow cytometry analysis was performed to characterize the neutrophils more accurately, and the data demonstrated considerably more $\mathrm{Gr} 1^{+} \mathrm{Ly}^{6} \mathrm{G}^{+}$cells (neutrophils) in BALF (Fig. 1E). As the first line of defense for the host, neutrophils contain diverse proteinases, including lactoferrin, serine protease and MPO, which can be rapidly released upon activation, and they also produce multiple pro-inflammatory cytokines, both contributing to tissue injury, including ALI (28). The present study detected the production of MPO in BALF, and the data indicated a much higher level of MPO in BALF compared with sham control mice (Fig. 1D). Therefore, the ALI mouse model demonstrated characteristic features of ALI, including high protein levels and neutrophil infiltration in BALF.

Protective effects of ethyl pyruvate on ALI. Ethyl pyruvate, a stable and simple lipophilic ester that derives from the 


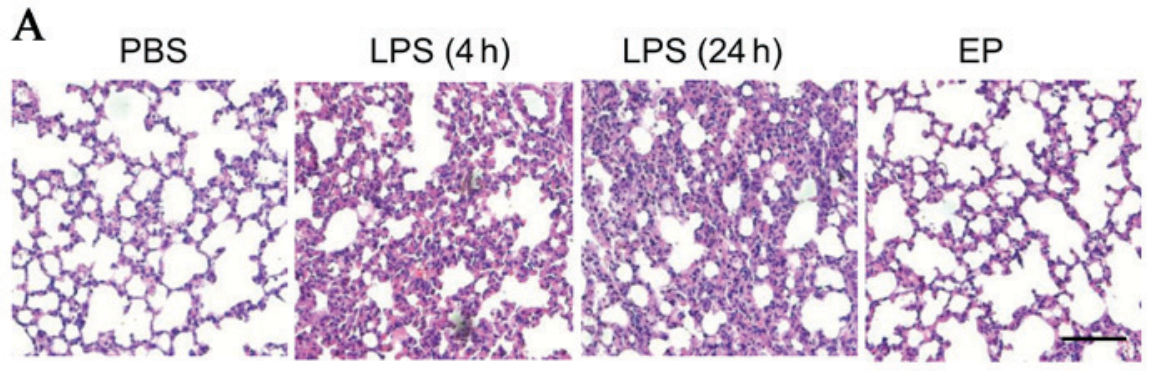

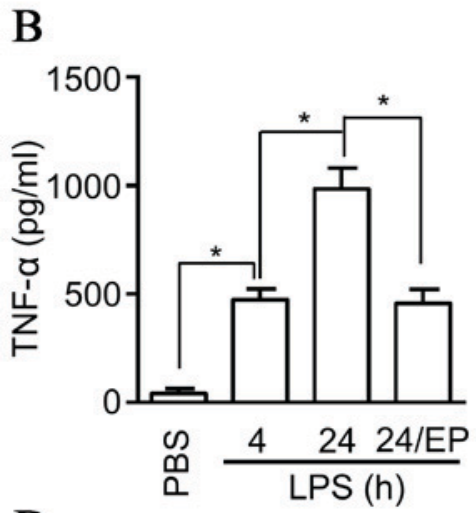

D

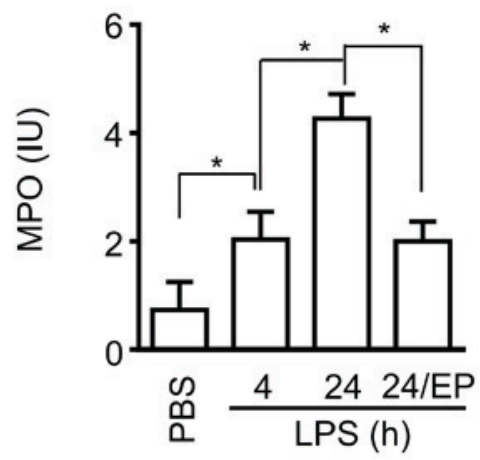

C

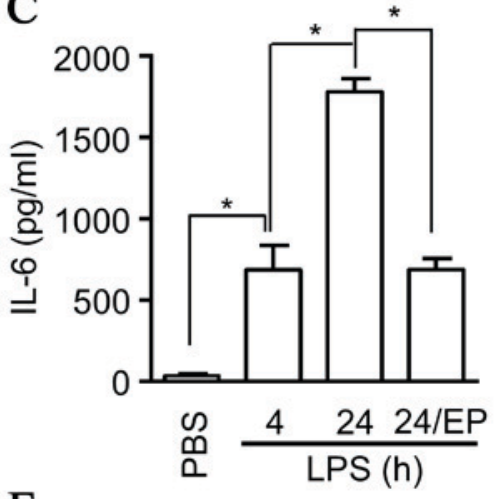

$\mathrm{E}$

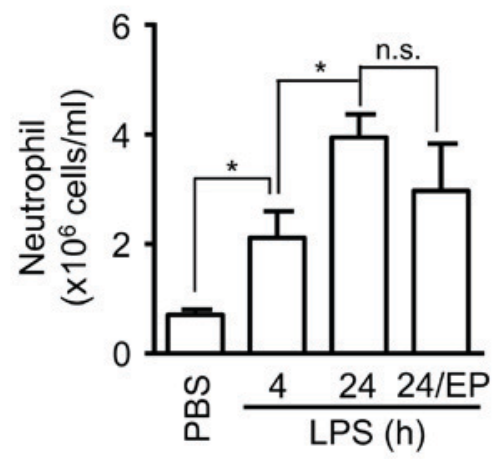

Figure 2. Protective effects of ethyl pyruvate on ALI. Eight-week old C57BL/6 male mice (6 mice per group) were administrated with ethyl pyruvate $(80 \mathrm{mg} / \mathrm{ml})$ or saline, then injected intratracheally with LPS (5 mg/kgs) at 4 or $24 \mathrm{~h}$. (A) Lungs samples were fixed, embedded in paraffin wax, sectioned at $5 \mu \mathrm{m}$ and stained with hematoxylin and eosin. The images are representative of three experiments (scale bar, $20 \mu \mathrm{m}$ ). BALF was collected from the mice and (B) TNF- $\alpha$, (C) IL-6, and (D) MPO were measured by ELISA. (E) The cells collected from BALF were stained with Gr1 and Ly6G ${ }^{+}$antibody, and were subsequently counted using flow cytometry. The data are presented as the mean \pm standard deviation of three independent experiments $\left({ }^{*} \mathrm{P}<0.05\right)$. ALI, acute lung injury; LPS, lipopolysaccharide; BALF, bronchoalveolar lavage fluid; TNF- $\alpha$, tumor necrosis factor- $\alpha$; IL-6, interleukin-6; MPO, myeloperoxidase; PBS, phosphate-buffered saline; n.s., non-significant.

endogenous metabolite pyruvate, has been demonstrated to protect against inflammation and to reduce organ dysfunction in several animal models of clinical illnesses, including burn injuries, severe sepsis and acute pancreatitis. Ethyl pyruvate has been demonstrated to exhibit a protective effect in ALI, probably through inhibition of the MAPK pathway in lungs. However, allowing for the multiple mechanisms of anti-inflammation, ethyl pyruvate contributes to protective effects against ALI through other pathways, remains to be elucidated. Following the application of ethyl pyruvate in ALI, an attenuation of ALI was observed, based on the inflammatory cell infiltration detected in H\&E staining (Fig. 2A), and decreased production of cytokines, including TNF- $\alpha$ (Fig. 2B), and IL-6 (Fig. 2C) in BALF from ALI mice compared with the saline group at 4 and $24 \mathrm{~h}$ (Fig. 3A). The production of MPO, which is mainly released from neutrophils, was also detected in BALF, and the results indicated a significant decrease of
MPO production in BALF (Fig. 2D). However, the infiltration of neutrophils in BALF exhibited no marked inhibition by ethyl pyruvate (Fig. 2E), suggesting that there exists other mechanisms for MPO release by neutrophils. Thus, the data suggested that ethyl pyruvate inhibits degranulation and attenuates ALI.

Enhanced autophagy in neutrophils in ALI mice model. Autophagy is a constitutive regulatory means of cellular homeostasis involved in diverse physiological and pathological events (10-15), and has been demonstrated to possess a pivotal role in neutrophil-mediated inflammation, thus the present study detected autophagy in neutrophils from BALF. Autophagy induction was assessed by monitoring LC3, as LC3 I conjugated to phosphatidylethanolamine (LC3 II), which links to autophagosome-membrane following the induction of autophagy. Following preparation from BAL fluid from mice, neutrophils were lysed and analyzed by immunoblotting for 


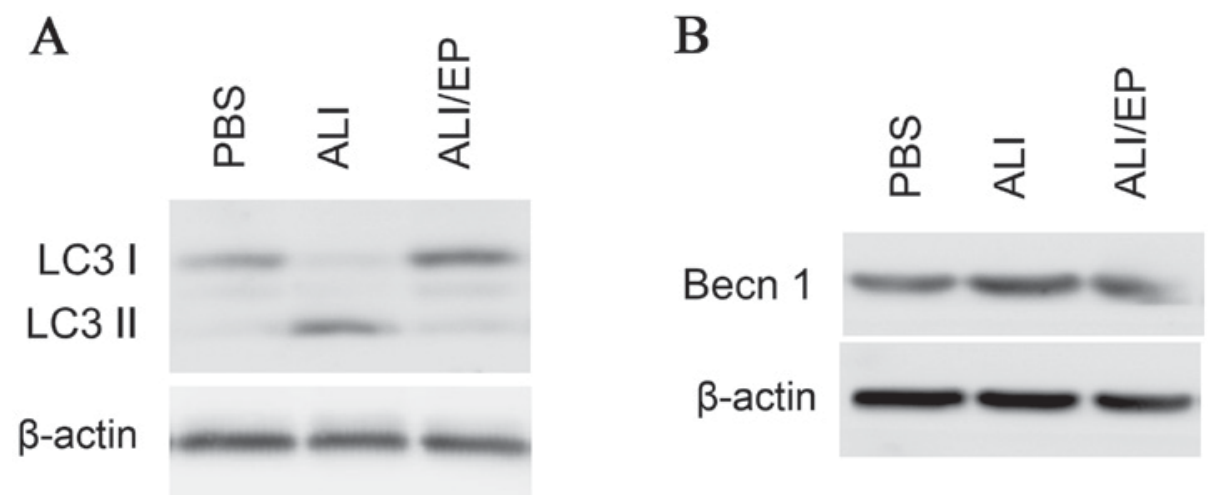

Figure 3. Enhanced autophagy in neutrophils in ALI mice model. Eight-week old C57BL/6 male mice (6 mice per group) were injected intratracheally with LPS $(5 \mathrm{mg} / \mathrm{kg})$ at 4 or $24 \mathrm{~h}$. Neutrophils from normal mice and LPS-induced ALI mice were isolated and lysed. Lysates were analyzed by immunoblotting with (A) LC3 antibodies for conversion of LC3-I to LC3-II and (B) Becn 1 antibodies. Images are representatives of three experiments. ALI, acute lung injury; LPS, lipopolysaccharide; LC3, microtubule-associated protein 1A/1B-light chain 3; EP, ethyl pyruvate.

LC3-II. LC3-II levels in neutrophils from mice with ALI were increased significantly compared with the control mice, while ethyl pyruvate reduced the autophagy (Fig. 3A). The expression levels of Beclin 1, a key regulator in autophagy, were monitored and the similar results to LC3 II were obtained (Fig. 3B). No significant differences were observed in the neutrophil amount present in BALF between the ALI group and the EP-treated group, while the cytokines, including TNF- $\alpha$ and IL-6, and particularly MPO, which are mainly released from neutrophils, are much higher in the BALF from ALI mice compared with the BALF from the EP-treated group. It was assumed that autophagy is important for neutrophil activation and degranulation, and ethyl pyruvate exerts protective effect in ALI by inhibiting neutrophil autophagy.

Ethyl pyruvate inhibits autophagy in neutrophils. To clarify the effects of autophagy on neutrophil degranulation, Atg5, a key regulator for autophagy, was knocked down with small interfering RNA in the neutrophil line, 32Dcl3 (Fig. 4A). Both LPS $(100 \mathrm{ng} / \mathrm{ml})$ and N-Formyl-Met-Leu-Phe (fMLP) $(200 \mathrm{nM})$ strongly induced neutrophil degranulation, as presented in Fig. 4B. However, the knockdown of Atg5 abolished the effect of LPS and fMLP on MPO release from neutrophils (Fig. 4D), emphasizing the essential role of autophagy for degranulation of neutrophils. 32Dcl3 cells were subsequently treated with LPS $(100 \mathrm{ng} / \mathrm{ml})$ and fMLP $(200 \mathrm{nM})$ for $14 \mathrm{~h}$ in the absence and presence of ethyl pyruvate, and the results demonstrated that ethyl pyruvate inhibited the induction of autophagy in 32Dcl3 cells (Fig. 4C). In addition, MPO release and TNF- $\alpha$ production by neutrophils were also decreased in the presence of ethyl pyruvate (Fig. 4D and E), consistent with the results of ATG5-knockdown treatment and confirming the role of autophagy in MPO release and TNF- $\alpha$ production by neutrophils. Together, the data illustrated the protective effect of ethyl pyruvate on ALI by inhibiting degranulation through blocking autophagy of neutrophils, unveiling a novel mechanism, which may lead to a novel therapeutic strategy for ALI.

\section{Discussion}

Neutrophils contribute to ALI through a number of mechanisms, including the generation of ROS due to excessive activation, the formation of neutrophil extracellular traps, the production of proinflammatory cytokines and the release of granule components. The evidence indicates that the biological alteration of neutrophils is involved in the initiation, development and resolution of ALI. Thus, therapy concentrated on inhibiting neutrophil activation and degranulation may provide one promising approach for ALI. The data from the present study demonstrated that neutrophils infiltrated into airspace during ALI experience increased autophagy, which is required for granule release, while ethyl pyruvate inhibited autophagy in neutrophils, and decreased granule release from neutrophils, thus attenuating lung injury in ALI. This novel mechanism clarifies the roles of ethyl pyruvate in ALI, and also provides a basis to develop a novel therapeutic approach, with autophagy as its target.

Ethyl pyruvate has been administrated in various animal models to ameliorate organ injury, including lung and hepatic injury $(4,5,29)$. It is widely accepted that ethyl pyruvate has an anti-inflammatory effect and decreases the production of cytokines, including TNF- $\alpha$, IL- 6 and high mobility group box 1 protein, thus reducing mortality. However, the cellular targets in these previous studies are elusive (30). In the present study, based on decreased MPO release, it was hypothesized that the neutrophil can be the cell type affected. Yet no significant difference in neutrophil infiltration between the control group and the ethyl pyruvate-treated group was observed, suggesting some other mechanism to regulate neutrophil biology. It was noticed that the results of the present study are inconsistent with the previous study by Kung et al (5). These inconsistences may be the result of different species of animal used and different methods, as Kung et al (5) used rats to construct their ALI model and counted all inflammatory cells instead of neutrophils.

As autophagy has been reported to serve an important role in neutrophil degranulation, autophagy in neutrophils isolated from BALF was identified and increased autophagy was observed, while ethyl pyruvate prevented the increase. In order to explore the underlying mechanism, 32Dcl3, a neutrophil cell line, was treated with LPS and enhanced autophagy and MPO release was observed, while the presence of ethyl pyruvate prevented the increase of autophagy. When Atg5 was knocked down, MPO release upon LPS stimulation was 

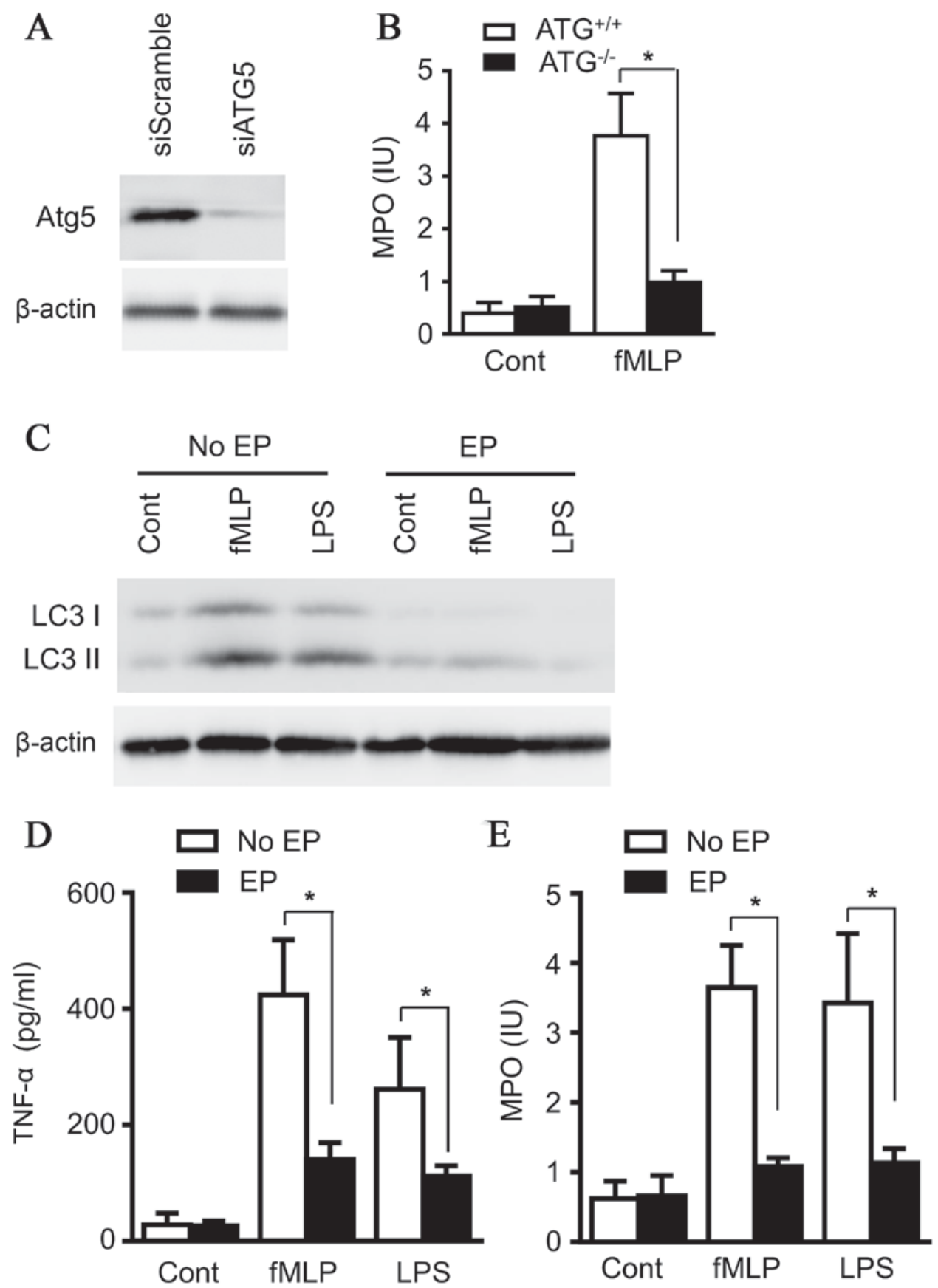

Figure 4. Ethyl pyruvate inhibits autophagy in neutrophils. (A) Murine myeloid cells, 32Dcl, 3 were transiently transfected with siScramble or siATG5 overnight, and the cells were lysed. Lysates were analyzed by immunoblotting with ATG5 antibody. (B) Murine myeloid cells, 32Dcl3, transiently transfected with siScramble or siATG5 overnight, were treated with fMLP $(200 \mathrm{nM})$ or LPS $(100 \mathrm{ng} / \mathrm{ml})$ for $14 \mathrm{~h}$, and supernatants from cultured neutrophils were collected and subjected to ELISA for detecting MPO. (C) Murine myeloid cells, 32Dcl3, were treated with fMLP (200 nM) or LPS (100 ng/ml) for $14 \mathrm{~h} \mathrm{in} \mathrm{the} \mathrm{absence}$ or presence of EP. Supernatants were collected and the cells were lysed. Lysates were analyzed by immunoblotting with LC 3 antibody. Images presented here were representatives of three experiments. Supernatants were subjected to ELISA for (D) TNF- $\alpha$ and (E) MPO. Images presented here were representatives of three experiments. The data are presented as the mean \pm standard deviation of three independent experiments ( $\mathrm{P}<0.05)$. ATG5, autophagy related 5; fMLP, N-Formyl-Met-Leu-Phe; LPS, lipopolysaccharide; MPO, myeloperoxidase; EP, ethyl pyruvate, LC3, microtubule-associated protein 1A/1B-light chain 3; TNF- $\alpha$, tumor necrosis factor- $\alpha$.

reduced, providing more evidence on the essential role of autophagy in neutrophil degranulation. Thus, using various methods, the present study emphasized the contribution of ethyl pyruvate in alleviating lung injury by dampening neutrophil autophagy.

In summary, the results revealed a novel mechanism of ethyl pyruvate in the alleviation of ALI by inhibiting autophagy in neutrophils and in turn dampening granule release; the results may also aid the identification of a potential therapeutic approach for ALI.

\section{Acknowledgements}

The present study was supported by the Shanghai Municipal Education Commission for Scientific Research in Outstanding Young Teachers of Universities in Shanghai, the National 
Health and Family Planning Commission of the People's Republic of China for 2013-2014 National Clinical Key Specialty Construction Project, and the Science and Technology Commission of Shanghai Municipality (grant no. 13ZR1426500).

\section{References}

1. Tsushima K, King LS, Aggarwal NR, De Gorordo A, D'Alessio FR and Kubo K: Acute lung injury review. Intern Med 48: 621-630, 2009.

2. Cui T, Miksa M, Wu R, Komura H, Zhou M, Dong W, Wang Z, Higuchi S, Chaung W, Blau SA, et al: Milk fat globule epidermal growth factor 8 attenuates acute lung injury in mice after intestinal ischemia and reperfusion. Am J Respir Crit Care Med 181: 238-246, 2010

3. Abraham E, Carmody A, Shenkar R and Arcaroli J: Neutrophils as early immunologic effectors in hemorrhage- or endotoxemia-induced acute lung injury. Am J Physiol Lung Cell Mol Physiol 279: L1137-L1145, 2000

4. Shang GH, Lin DJ, Xiao W, Jia CQ, Li Y, Wang AH and Dong L: Ethyl pyruvate reduces mortality in an endotoxin-induced severe acute lung injury mouse model. Respir Res 10: 91, 2009.

5. Kung CW, Lee YM, Cheng PY, Peng YJ and Yen MH: Ethyl pyruvate reduces acute lung injury via regulation of iNOS and HO-1 expression in endotoxemic rats. J Surg Res 167: e323-e331, 2011.

6. Matute-Bello G, Frevert CW and Martin TR: Animal models of acute lung injury. Am J Physiol Lung Cell Mol Physiol 295: L379-L399, 2008.

7. Matthay MA and Howard JP: Progress in modelling acute lung injury in a pre-clinical mouse model. Eur Respir J 39: 1062-1063, 2012 .

8. Chopra M, Reuben JS and Sharma AC: Acute lung injury:Apoptosis and signaling mechanisms. Exp Biol Med (Maywood) 234: 361-371, 2009.

9. Brinkmann V and Zychlinsky A: Neutrophil extracellular traps: Is immunity the second function of chromatin? J Cell Biol 198 773-783, 2012.

10. Singh R and Cuervo AM: Autophagy in the cellular energetic balance. Cell Metab 13: 495-504,2011.

11. Mizushima N and Komatsu M: Autophagy: Renovation of cells and tissues. Cell 147: 728-741, 2011.

12. Cuervo AM: Cell biology. Autophagy's top chef. Science 332: 1392-1393, 2011.

13. Shanware NP, Bray K and Abraham RT: The PI3K, metabolic, and autophagy networks: Interactive partners in cellular health and disease. Annu Rev Pharmacol Toxicol 53: 89-106, 2013.

14. Patel KK and Stappenbeck TS: Autophagy and intestinal homeostasis. Annu Rev Physiol 75: 241-262, 2013.
15. Choi AM, Ryter SW and Levine B: Autophagy in human health and disease. New Engl J Med 368: 651-662, 2013.

16. Netea-Maier RT, Plantinga TS, Van De Veerdonk FL, Smit JW and Netea MG: Modulation of inflammation by autophagy: Consequences for human disease. Autophagy 12: 245-260, 2016.

17. Xu C, Feng K, Zhao X, Huang S, Cheng Y, Qian L, Wang Y, Sun H, Jin M, Chuang TH and Zhang Y: Regulation of autophagy by E3 ubiquitin ligase RNF216 through BECN1 ubiquitination. Autophagy 10: 2239-2250, 2014.

18. Xu Y, Jagannath C, Liu XD, Sharafkhaneh A, Kolodziejska KE and Eissa NT: Toll-like receptor 4 is a sensor for autophagy associated with innate immunity. Immunity 27: 135-144, 2007.

19. Nathan C: Secretory products of macrophages: Twenty-five years on. J Clin Invest 122: 1189-1190, 2012.

20. Sica A and Mantovani A: Macrophage plasticity and polarization: In vivo veritas. J Clin Invest 122: 787-795, 2012.

21. Petrovski G, Ayna G, Majai G, Hodrea J, Benko S, Mádi A and Fésüs L: Phagocytosis of cells dying through autophagy induces inflammasome activation and IL-1 $\beta$ release in human macrophages. Autophagy 7: 321-330, 2011.

22. Gordon S and Martinez FO: Alternative activation of macrophages: Mechanism and functions. Immunity 32: 593-604, 2010.

23. Auffray C, Sieweke MH and Geissmann F: Blood monocytes: Development, heterogeneity and relationship with dendritic cells. Annu Rev Immunol 27: 669-692, 2009.

24. Bhattacharya A, Wei Q, Shin JN, Abdel Fattah E, Bonilla DL, Xiang Q and Eissa NT: Autophagy is required for neutrophil-mediated inflammation. Cell Rep 12: 1731-1739, 2015.

25. Xu C, Liu J, Hsu LC, Luo Y, Xiang R and Chuang TH: Functional interaction of heat shock protein 90 and Beclin 1 modulates Toll-like receptor-mediated autophagy. FASEB J 25: 2700-2710, 2011.

26. Wysocki M, Cracco C, Teixeira A, Mercat A, Diehl JL, Lefort Y, Derenne JP and Similowski T: Reduced breathing variability as a predictor of unsuccessful patient separation from mechanical ventilation. Crit Care Med 34: 2076-2083, 2006.

27. Strait RT, Hicks W, Barasa N, Mahler A, Khodoun M, Köhl J, Stringer K, Witte D, Van Rooijen N, Susskind BM and Finkelman FD: MHC class I-specific antibody binding to nonhematopoietic cells drives complement activation to induce transfusion-related acute lung injury in mice. J Exp Med 208: 2525-2544, 2011

28. Aimone JB, Li Y, Lee SW, Clemenson GD, Deng W and Gage FH: Regulation and function of adult neurogenesis: From genes to cognition. Physiol Rev 94: 991-1026, 2014.

29. Shen M, Lu J, Dai W, Wang F, Xu L, Chen K, He L, Cheng P, Zhang Y, Wang C, et al: Ethyl pyruvate ameliorates hepatic ischemia-reperfusion injury by inhibiting intrinsic pathway of apoptosis and autophagy. Mediators Inflamm 2013: 461536, 2013.

30. Davé SH, Tilstra JS, Matsuoka K, Li F, DeMarco RA, Beer-Stolz D, Sepulveda AR, Fink MP, Lotze MT and Plevy SE: Ethyl pyruvate decreases HMGB1 release and ameliorates murine colitis. J Leukoc Biol 86: 633-643, 2009. 